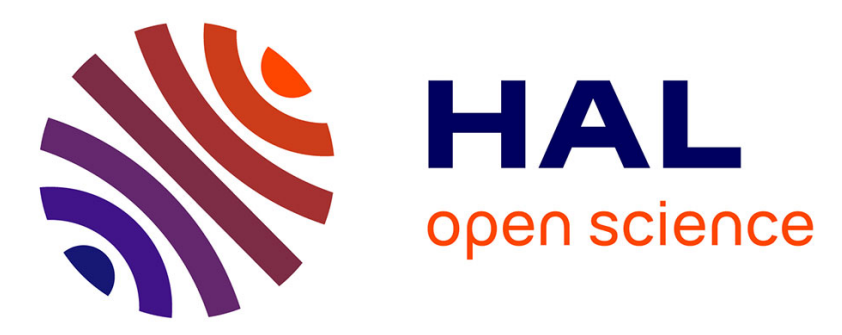

\title{
Identification de la nature de milieux stratifiés à partir de l'écho renvoyé
}

A. Djellouli, B. Latu, J. Trouilhet

\section{To cite this version:}

A. Djellouli, B. Latu, J. Trouilhet. Identification de la nature de milieux stratifiés à partir de l'écho renvoyé. Journal de Physique IV Proceedings, 1994, 04 (C5), pp.C7-1075-C7-1078. 10.1051/jp4:19945236 . jpa-00252923

\section{HAL Id: jpa-00252923 https://hal.science/jpa-00252923}

Submitted on 1 Jan 1994

HAL is a multi-disciplinary open access archive for the deposit and dissemination of scientific research documents, whether they are published or not. The documents may come from teaching and research institutions in France or abroad, or from public or private research centers.
L'archive ouverte pluridisciplinaire HAL, est destinée au dépôt et à la diffusion de documents scientifiques de niveau recherche, publiés ou non, émanant des établissements d'enseignement et de recherche français ou étrangers, des laboratoires publics ou privés. 


\title{
Identification de la nature de milieux stratifiés à partir de l'écho renvoyé
}

\author{
A. DJELLOULI, B. LATU et J.F. TROUILHET
}

Laboratoire d'Acoustique de Métrologie d'Instrumentation, 38 rue des 36 Ponts, 31400 Toulouse, France

\begin{abstract}
Absorbing materials modelisation as received much recent attention including domains like : aéro-acoustic (identification and classification of different materials,...) underwater acoustic (various types of sea beds, hydrobiology,...).

We present in this paper two methods to estimating pertinent parameters from material reflections :

- reflector response when a sinusoidal excitation is applied (narrow band).

- impulse response of stratified environments (wide band) .
\end{abstract}

\section{INTRODUCTION}

La première partie consiste à présenter la reconnaissance des réflecteurs de nature différente qui se décompose en deux étapes :

- Modélisation de l'écho rétro-diffusé par le matériau pour en extraire l'information pertinente.

- Classification des différents réflecteurs à partir des paramètres issus de la modélisation.

La deuxième partie a pour objectif de mettre au point une méthode d'analyse de la réponse impulsionnelle des milieux stratifiés. La méthode d'analyse choisie est une transformée temps-fréquence.Dans un premier temps nous avons utilisé un modèle mathématique, qui par la suite a été validé par l'expérimentation.

\section{REPONSE A UN TRAIN DE SINUSOIDE}

Un signal de type porteuse, modulé par une information bande étroite,est émis au travers d'un émetteur ultrasonore en incidence normale (fig2.1) par rapport au reflecteur.

Toute l'information propre au réflécteur est contenue dans l'enveloppe temporelle de l'écho renvoyé (fig2.2). Notre objectif consiste à modéliser cette enveloppe en vue d'une classification des différents réflecteurs.

\subsection{Modélisation}

Le but d'une modélisation consiste à déterminer un modèle mathématique qui permette de résumer l'information caractéristique du signal réfléchi en un nombre fini de paramètres. Toutefois, il est clair que la pertinence du modèle retenu permettra d'extraire un ensemble de coefficients qui peut ne pas garantir une bonne classification.

Nous avons testé en premier lieu les méthodes paramétriques classiques telles que la modélisation autorégressive AR et la modélisation de Prony [1] qui permettent d'aboutir à une analyse spectrale de l'enveloppe. Les essais de modélisation sur des matériaux aussi différents que l'aluminium et la laine de verre n'ont pas fait apparaitre de différences suffisamment significatives sur les paramètres du modèle pour envisager une classification à partir de ceux ci.

A partir de l'allure temporelle de l'enveloppe suffisamment caractéristique de chaque matériau, nous avons cherché dans un deuxième temps à utiliser cette propriété à des fins classifiantes. Ainsì, une modélisation liée à l'énergie de l'écho a été mise en oeuvre. Pour cela nous avons segmenté le signal en trois zones élémentaires (fig 2.3), et calculé dans chacune d'elles les pentes $\mathrm{A} 1, \mathrm{~A} 2$, et $\mathrm{A} 3$. Les instants de segmentation $\mathrm{t} 1$, $\mathrm{t} 2$, et $\mathrm{t} 3$ corespondent respectivement aux intersections des axes : (axe $t, A 1),(\mathrm{A} 1, \mathrm{~A} 2)$ et $(\mathrm{A} 2, \mathrm{~A} 3)$.

Le vecteur représentatif de chaque matériau est: $\mathrm{P}=[\mathrm{t} 1 \mathrm{t} 2 \mathrm{t} 3 \mathrm{~A} 1 \mathrm{~A} 2 \mathrm{~A} 3]$ 


\subsection{Classification}

\subsubsection{Prétraitement des paramètres}

Afin d'assurer l'efficacité de la classification on cherche à déterminer les paramètres les plus pertinents en éliminant ceux qui sont corrélés (de même un paramètre non significatif peut nuire à une bonne classification ).

Nous avons utilisé le critère de Fisher : Il classe les paramètres par ordre de pertinence en se basant sur un rapport dont le numérateur reflète l'éloignement entre les classes et le dénominateur le regroupement des classes sur elles mêmes.

\subsubsection{Algorithme des centres mobiles}

Cette méthode rentre dans le cadre de la classification en mode non supervisé. L'algorithme des centres mobiles [2] a pour but de construire une partition de l'ensemble étudié, en initialisant le nombre de classes $\mathrm{N}_{\mathrm{c}}$ et la partition. Soit par une connaissance à priori des classes ou par une partition au hasard des individus sur ces classes. On exécute ensuite les opérations suivantes :

(a)-Pour chaque classe $\mathrm{C}_{\mathrm{k}}$ déterminer le centre de gravité gk.

(b)-Réafecter chaque individu $\underline{x}_{i}$ à la classe $\mathrm{Ck}$ dont le centre de gravité est le plus proche

au sens de la distance euclidienne.

(c)-Retoumer en (a) tant que des modifications dans la composition des classes surviennent.

Une bonne classification doit minimiser le moment intraclasse pour fournir les classes les plus condensées que possible ou maximiser le moment interclasse pour avoir des classes bien éloignées.

L'inconvénient majeur de cette méthode est que l'on n'a pas la certitude d'obtenir la meilleure solution. Pour pallier à ce problème on exécute plusieurs fois l'algorithme complet avec des partitions initiales différentes. On retient alors celle qui minimise le moment intraclasse.

\subsection{Résultats pratiques}

Le signal émis est un train de sinusoïde modulé en amplitude, de fréquence $38,5 \mathrm{khz}$ et de largeur $0,6 \mathrm{~ms}$. On échantilonne à $125 \mathrm{khz}$. Trois matériaux différents ont été utilisés: Aluminium (réflecteur dur), Moquette épaisse (réflecteur moyen) et Laine de verre (réflecteur mou) à raison de dix acquisitions différentes par matériau. L'enveloppe temporelle est calculée à partir du signal analytique.

Tableau-1- distance entre classes

\begin{tabular}{|c|c|c|c|}
\hline $\mathrm{n}^{\circ}$ classe & 1 & 2 & 3 \\
\hline 1 & 0.00 & 222.10 & 110.45 \\
\hline 2 & 22.10 & 0.00 & 644.23 \\
\hline 3 & 110.45 & 644.23 & 0.00 \\
\hline
\end{tabular}
Aprés modélisation de l'enveloppe, la pertinence des paramètres est évolué par le critère de Fisher. Il en résulte que les paramètres $\mathrm{P} 4$ et $\mathrm{P} 6$ qui sont respectivement les pentes montante et descendante de l'enveloppe sont de loin les plus significatifs pour notre étude.

La classification des matériaux selon les paramètres P4 et P6 par l'algorithme des centres mobiles permet de séparer idéalement les trois classes (aluminium, moquette, laine de verre), avec des taux de réussite de $100 \%$ (fig.2.4). Les distances entre classes sont données dans le tableau.1.

\section{MODELISATION ET ANALYSE DE LA REPONSE IMPULSIONNELLE}

Nous souhaitons pouvoir déterminer la nature d'un milieu stratifié par l'utilisation de la transformée en ondelettes continues. Pour pouvoir interpréter les calculs, nous nous appuyons sur un modèle mathématique introduit par K.STEPHANAKIS [3], visant à déterminer la réponse impulsionnelle d'un tel milieu.

\subsection{Modélisation de la réponse impulsionnelle}

L'idée consiste à considérer les trajets multiples d'une onde acoustique se propageant dans un milieu stratifié avec une incidence normale. En considérant chaque strate du matériau comme composée d'une succession de couches fictives, tels que le temps de propagation d'un aller retour de l'onde dans chacune d'elles soit égale à l'unité, cela permet d'avoir une épaisseur de strates quelconque.Pour chaque interface fictive, le coefficient de réflexion est nul. Les hypothèses supplémentaires du modèle se résument à considérer un milieu non dispersif, non absorbant et assimilable à un filtre linéaire.

Les relations entre les ondes transmises $d_{j}(t)$ et réfléchies $u_{j}(t)$ de la couche $j$, en terme de coéfficient de réflexion $\mathrm{r}_{\mathrm{j}}$ peuvent se décrire à l'interface $\mathrm{j}$ suivant :

$$
d_{j+1}=\left(1+r_{j}\right) d_{j}(t-0,5)-r_{j} u_{j+1}(t) \quad u_{j}(t+0,5)=r_{j} d_{j}(t-0,5)+\left(1-r_{j}\right) u_{j+1}(t)
$$

Le passage à la transformée en $z$ et le développement de la reccurence sur chaque interface permet d'aboutir à la fonction de transfert du milieu stratifié par:

$$
\frac{u_{0}(z)}{d_{0}(z)}=\frac{Q_{k}(z)-r_{0} P_{k}(z)}{P_{k}(z)-r_{0} Q_{k}(z)}
$$


Où les polynomes $P_{k}(z)$ et $Q_{k}(z)$ s'écrivent selon :

$$
\begin{aligned}
& P_{k+1}(z)=P_{k}(z)-r_{k+1} \cdot z^{k+1} \cdot Q_{k}\left(z^{-1}\right) \\
& Q_{k+1}(z)=Q_{k}(z)-r_{k+1} \cdot z^{k+1} \cdot P_{k}\left(z^{-1}\right) \\
& P_{0}(z)=P_{0}\left(z^{-1}\right)=1 \\
& Q_{0}(z)=Q_{0}\left(z^{-1}\right)=-r_{0}
\end{aligned}
$$

\subsection{Validations expérimentales}

Pour valider le modèle précédent nous avons réalisé des acquisitions selon la fig(3.1) et la fonction de transfert est obtenue par le rapport entre le signal reçu (micro 2) et le signal émis (micro 1).

\subsection{Analyse de la réponse impulsionnelle}

Dans un but d'analyse de différentes couches de milieu stratifié (identification de la nature et de l'épaisseur de chaque couche), nous souhaitons exploiter les propriétés de la décomposition de la transformée en ondelettes à partir d'une fonction mère selon les opérateurs de dilatation et de décalage. Pour cela il est essentiel de choisir une ondelette qui puisse exiber les réponses impulsionnelles de chaque milieu réfléchissant. Notre choix s'est porté sur l'ondelette de Morlet définie par :

$$
\psi(\mathrm{t})=\exp \left(\mathrm{jw}_{0} \mathrm{t}-\mathrm{t}^{2} / 2\right) \quad \mathrm{w}_{0}=\Pi \sqrt{2 / \log 2}
$$

w0 fixe le nombre d'oscillations dans le support effectif de la gaussiene. Le calcul de la transformée à

$$
\text { l'échelle a et au décalage } b \text { s'écrit : } \quad w_{x}(a, b)=\frac{1}{\sqrt{a}} \int_{R} x(t) \Psi^{*}(t-b / a) d t
$$

\subsection{Résultats}

La simulation d'un milieu stratifié comportant deux couches physiques conduit à la réponse impulsionnelle illustrée fig(3.a) et sa transformée en ondelettes fig(3.b). La mesure de la réponse impulsionnelle d'un milieu air / eau / métal est illustrée fig(4.a) et sa transformée en ondelettes fig(4.b).

Sur la figure (3.b), on peut discerner les maxima de la transformée dont la localisation temporelle indique les changements de couches du milieu. Toutefois il reste difficile de déduire la nature de la couche (coefficient de réflexion) uniquement par l'amplitude de ces maxima. La simulation sur des milieux réels fig(4.a) et fig(4.b) tels que air / métal, air / bois, ...montre que la position en échelle des maxima de la transformée reste difficile à interpréter quant à la nature du matériau (12.10 métal, 15.6 bois,...).

Toutefois comme pour les signaux simulés la localisation temporelle des maxima de la transformée en ondelettes indique les changements de strates (air / eau et eau / métal). Sur la fig(4.b) cette séparation temporelle est bien proportionelle au temps de propagation de l'onde acoustique dans chacune de ces couches.

\section{CONCLUSION}

La méthode bande étroite donne d'excellents résultats pour des manipulations où l'on maitrise les conditions, telles que en aéroacoustique (présenté au \$ 2). Cependant il est à prévoir que les résultats se dégraderont lors d'expérimentation en milieu naturel. Pour cela la méthode large bande, bien que plus complexe à mettre en oeuvre devrait permettre de meilleurs résultats. En l'état actuel de nos travaux la phase d'extraction des paramètres n'a pas pu être faite de façon systématique. Nous espérons réaliser dans un proche avenir la comparaison de ces deux techniques pour la classification de réflécteurs issus de milieux naturels.

\section{Bibliographie}

[1] S.M.KAY \& S.L MARPLE, "Spectrum Analysis-a modern perspective".Proc of IEEE,69 n 11, nov 1981, 1380 -1419.

[2] J.F.TROUILHET, "Etude et réalisation d'un dispositif de reconnaissance automatique de signature acoustique ".Thèse de doctorat de l'INP de Toulouse juin 1992.

[3] K.STEPHANAKIS, "Identification des facteurs de réflexion d'un milieu stratifié par les techniques de traitement du signal". Thèse de la faculté des sciences de l'Université de Provence février 1977.

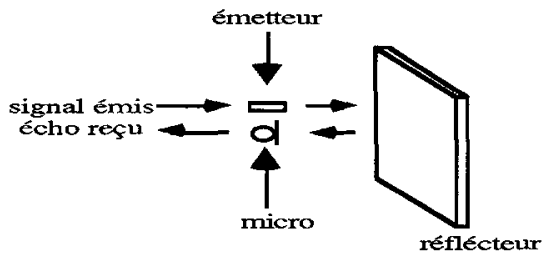




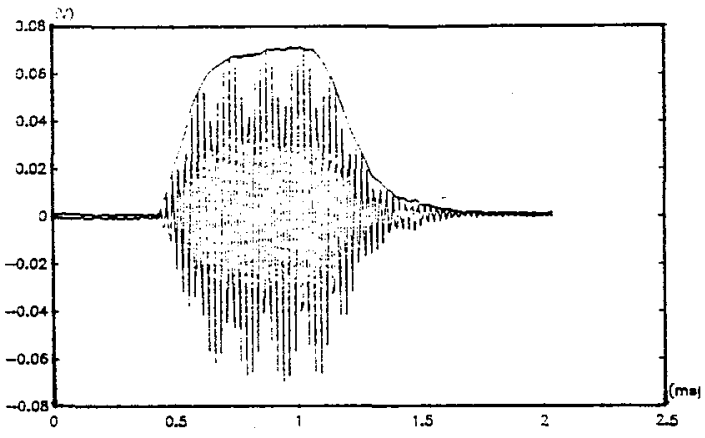

Fig-2.2- Echo renvoyé et son Enveloppe

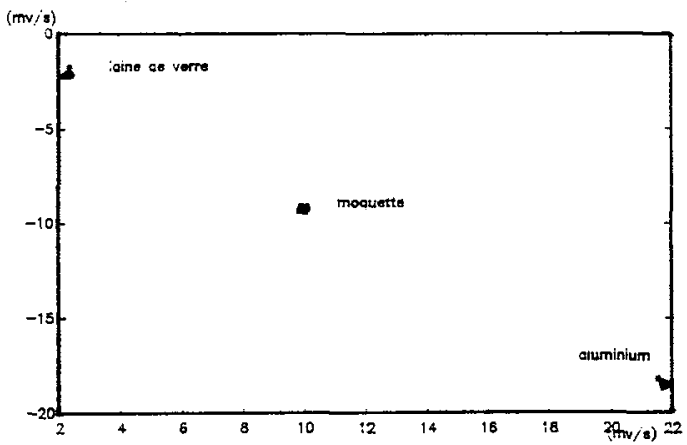

Fig-2.4- Distance entre classes

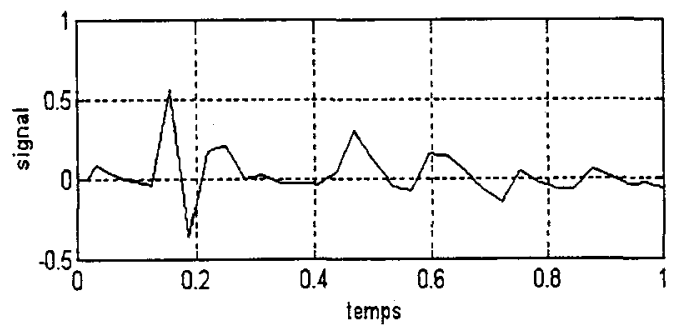

Fig-3.a- Réponse impulsionnelle simulée

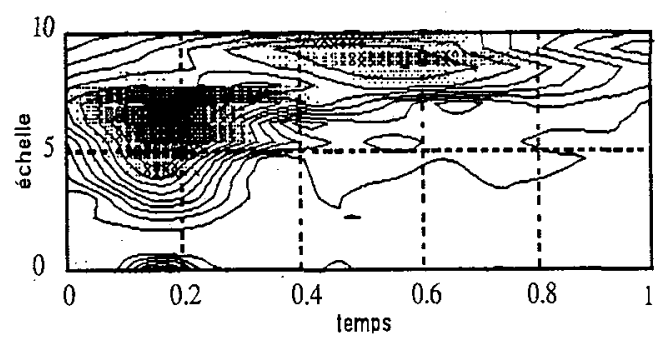

Fig-3.b- Transformée en ondelettes

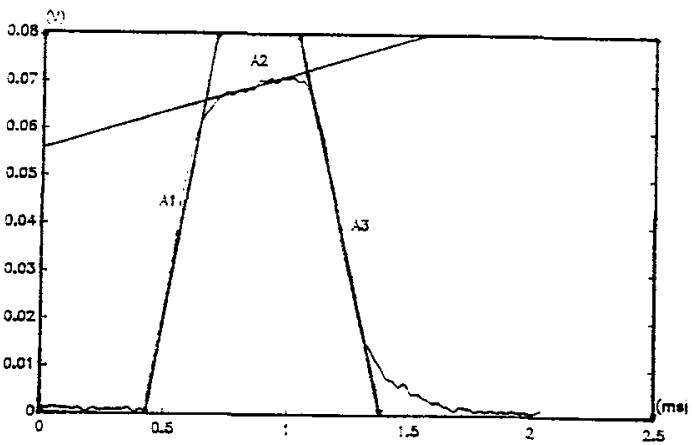

Fig-2.3- Modélisation de l'enveloppe

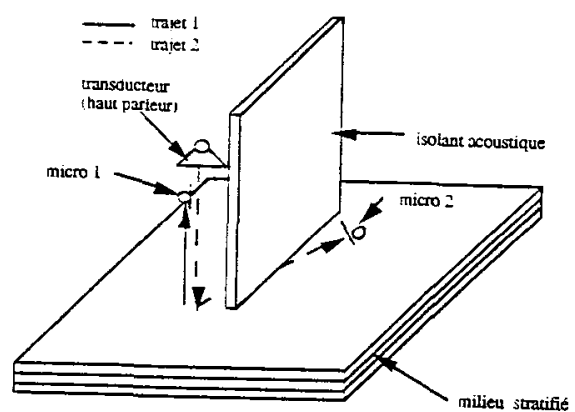

Fig-3.1- Expérimentation large bande

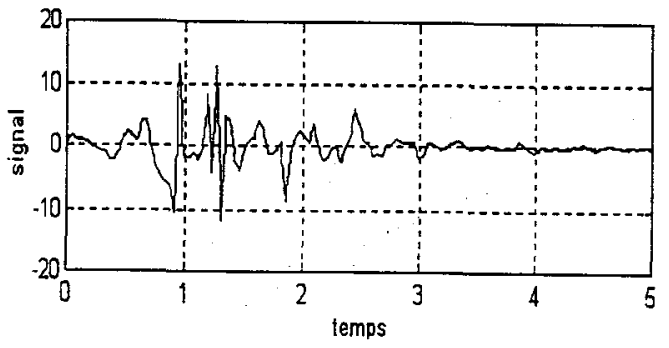

Fig-4.a- Réponse impulsionnelle mesurée

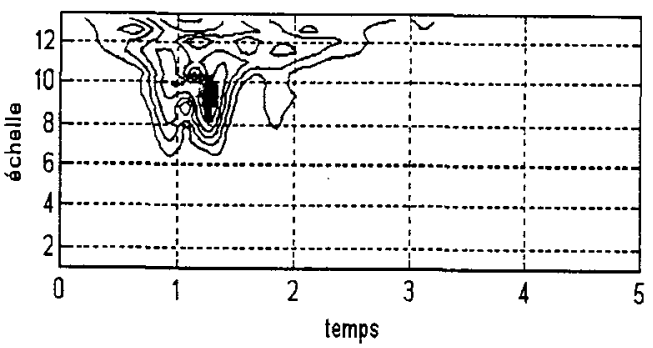

Fig-4.b- Transformée en ondelettes 